\title{
Electronic structure and magnetic properties of cobalt intercalated in graphene on $\operatorname{Ir}(111)$
}

\author{
H. Vita, ${ }^{1}$ St. Böttcher, ${ }^{1}$ P. Leicht, ${ }^{2}$ K. Horn, ${ }^{1}$ A. B. Shick, ${ }^{3}$ and F. Máca ${ }^{3}$ \\ ${ }^{1}$ Fritz-Haber-Institut der Max-Planck-Gesellschaft, Department of Physical Chemistry, Faradayweg 4-6, 14195 Berlin, Germany \\ ${ }^{2}$ Universität Konstanz, Fachbereich Physik, Universitätsstrasse 10, 78457 Konstanz, Germany \\ ${ }^{3}$ Institute of Physics ASCR, Na Slovance 2, 18221 Prague, Czech Republic
}

(Received 22 August 2014; published 22 October 2014)

\begin{abstract}
Using a combination of photoemission and x-ray magnetic circular dichroism (XMCD), we characterize the growth and the electronic as well as magnetic structure of cobalt layers intercalated in between graphene and $\operatorname{Ir}(111)$. We demonstrate that magnetic ordering exists beyond one monolayer intercalation, and determine the Co orbital and spin magnetic moments. XMCD from the carbon edge shows an induced magnetic moment in the graphene layer, oriented antiparallel to that of cobalt. The XMCD experimental data are discussed in comparison to our results of first-principles electronic structure calculations. It is shown that good agreement between theory and experiment for the Co magnetic moments can be achieved when the local-spin-density approximation plus the Hubbard $U(\operatorname{LSDA}+U)$ is used.
\end{abstract}

DOI: 10.1103/PhysRevB.90.165432

PACS number(s): 75.75.-c, 75.30.Gw, 79.60.-i

\section{INTRODUCTION}

Interaction of graphene with transition metal surfaces has attracted much interest. First, these surfaces are ideal templates for the growth of high quality films, and their use in this context is seen as a pathway towards mass production of large scale transferable graphene [1,2]. More importantly, from a point of view of the basic physical properties and applications, the ferromagnetic substrates such as $\mathrm{Ni}(111)$ and $\mathrm{Co}(0001)$ are interesting, since they induce the magnetic polarization in graphene [2-4]. When projected onto the hexagonal surface, only the spin-minority states of the transition metals overlap with a graphene valence state at the $K$ point of the Brillouin zone (BZ) near the Fermi level. Graphene can thus act as a spin filter, and a sizable difference in spin-dependent transmission has been predicted [5]. We have shown earlier that the proximity of graphene to the ferromagnetic $\mathrm{Ni}(111)$ substrate induces a sizable magnetic moment in the carbon $\pi$ states as determined from $\mathrm{C} K$ edge $\mathrm{x}$-ray circular magnetic dichroism [2,3], strengthened when a monolayer of iron is intercalated between the $\mathrm{Ni}$ substrate and the graphene layer [4].

An alternative route towards inducing magnetic moments in graphene uses magnetic intercalates between graphene and a nonmagnetic substrate [6]. Here we study the influence of thin layers of ferromagnetic Co sandwiched between the nonmagnetic transition-metal substrate $[\operatorname{Ir}(111)]$ and a graphene overlayer. We are thus able to examine an emerging ferromagnetic behavior in a thin cobalt film from the monolayer upwards, in a morphology that suppresses islanding, and to study the transfer of magnetic moment from the metal onto the carbon $\pi$ states. We show the emergence of ferromagnetic behavior of Co beyond the first monolayer, and obtain excellent agreement on the spin and orbital magnetic moment by comparison with state-of-the art density-functional theory (DFT) calculations. We also provide an in-depth study of the growth and intercalation process using core and valence level photoemission and low energy electron diffraction.

The paper is organized as follows: the experimental methods are presented first, followed by the results for the characterization of Co film growth and intercalation and the characterization of the valence electronic structure by photoelectron spectroscopy. The determination of the magnetic properties by magnetic circular dichroism (XMCD) comes next, followed by a brief discussion. The theory part begins with a description of the methods to obtain the structural optimization, and the approaches to calculate the magnetic properties of intercalated monolayers and double layers. The paper ends with a comparison of theoretical and experimental results and a general discussion.

\section{EXPERIMENTAL METHODS AND RESULTS}

\section{A. Methods}

The experiments were performed at beamline D1011 of MAX-lab, Lund, Sweden, and at beamline UE56/2-PGM1 at BESSY II, Berlin, Germany. The sample temperature was kept at $T=90 \mathrm{~K}$ in all measurements and during Co deposition, with a base pressure in the experimental station not exceeding $1 \times 10^{-10} \mathrm{mbar}$. X-ray absorption spectroscopy (XAS) and XMCD spectra were collected at both the Co $L_{2,3}$ and the $\mathrm{C} K$ absorption edges in partial electron yield (PEY) and total electron yield (TEY) mode with an energy resolution of $100 \mathrm{meV}$. Magnetic dichroism spectra were obtained with circularly polarized light (degree of polarization $P=75 \%$ ) at different angles in the remanent magnetic state of the graphene/Co/Ir(111) system, after applying a magnetic field of 500 Oe along the out-of-plane direction. Angle-resolved photoemission spectroscopy measurements were performed at photon energies of $h v=65 \mathrm{eV}$ and $h v=94 \mathrm{eV}$ respectively, core-level photoemission spectroscopy at $h v=400 \mathrm{eV}$. The photoemission station employs a PHOIBOS-100 hemispherical analyzer from SPECS GmbH and a five-axis motorized manipulator, allowing a precise alignment of the sample in $k$ space. The sample was azimuthally pre-aligned along the $\Gamma-K$ direction of the graphene-derived Brillouin zone with the angle dispersive direction on the channelplate images acquired perpendicular to $\Gamma-K$. Three-dimensional (3D) data sets of the photoemission intensity were obtained by tilting the sample systematically along the horizontal axis. Each voxel in the 3D-data sets thus contains the photoemission intensity as a 
function of kinetic energy and two emission angles converted to reciprocal space, $I\left(E_{\mathrm{kin}}, k_{x}, k_{y}\right)$.

Prior to preparation of graphene on $\operatorname{Ir}(111)$, we used a procedure of repeated cycles of annealing in an oxygen atmosphere (partial pressure $1 \times 10^{-7}$ mbar at $1200 \mathrm{~K}$ ) and flashing (up to $1900 \mathrm{~K}$ ) to obtain a clean $\operatorname{Ir}(111)$ crystal. Subsequently, the graphene layer on $\operatorname{Ir}(111)$ was prepared via cracking of propylene gas (partial pressure $1 \times 10^{-7} \mathrm{mbar}$ at $1600 \mathrm{~K}$ ) yielding high quality single-phase $R 0^{\circ}$ graphene on $\operatorname{Ir}(111)$ [7]. The quality of the graphene/Ir(111) sample was checked by low energy electron diffraction (LEED), indicating a hexagonal pattern with sharp main spots and additional satellite spots due to the moiré structure [8]. XPS spectra of the $\mathrm{C} 1 s$ core level reveal the high quality of the graphene/Ir(111) sample by a sharp single component line with no additional peaks at higher binding energies [Fig. 1(a)].

The methods used to calculate the electronic structure and the magnetic moments of the intercalated Co layers are described in the Theory section.

\section{B. Results}

Intercalation of various species is a well-known method to modify the properties of epitaxial graphene on metals and semiconductors [9-13]. In the case of graphene on metals, the ability of intercalated chemical species to decouple graphene from the $\mathrm{Ni}(111)$, recovering the Dirac fermion character of its collective excitations (phonon [9,14-16] and plasmon [17,18] modes) and its valence band [10,19], was already discovered [12] before the current rush of publications.

In the case of graphene on silicon carbide, for example, intercalation of hydrogen leads to "quasi-free-standing" graphene, reducing the detrimental effect of the so-called buffer layer on the carrier mobility [20].

For the present case of Co intercalation in graphene/Ir(111) [21-24], a considerable lattice mismatch occurs, similar to the case of an intercalated Ni layer [25], which on the one hand gives the opportunity to examine the electronic structure of thin metallic films under tensile stress, but also necessitates a close examination of the growth mode at the monolayer stage and beyond. Here we use C $1 s$ and Ir $4 f$ core-level photoemission to study the thickness and temperature dependence of Co interaction with graphene/Ir(111).

Photoelectron spectra in [Fig. 1(a)] show the C $1 s$ core level for different steps of sample preparation, starting from the pristine graphene/Ir(111) (black), to deposited $\mathrm{Co} /$ graphene/Ir(111) (blue), and finally intercalated graphene/Co/Ir(111) (green) system. The initial C $1 s$ corelevel spectra show a single component peak at $284.17 \mathrm{eV}$ which is reduced in intensity upon Co deposition on top of graphene up to a coverage of two monolayers' equivalent. Because of the low sample temperature, we assume that the film grows in a disordered layerwise manner. An additional broad peak around $283.00 \mathrm{eV}$ can be related to the formation of a surface Co-C carbide [26]. Intercalation of the twomonolayer (ML)-thick Co layer underneath graphene was performed via stepwise annealing of the deposited layer of Co on graphene/ $\operatorname{Ir}(111)$. In this procedure, the intensity of the $\mathrm{C} 1 s$ and Ir $4 f$ photoelectron spectra, observed in real time, was taken as evidence to discover when the temperature window required for successful intercalation is reached [Figs. 1(b) and 1(d)]. This method permits to carefully control the formation of an intercalated Co film underneath graphene/Ir(111).

Upon intercalation, the Co-C carbide peaks vanishes and the main $\mathrm{C} 1 s$ peak is strongly shifted towards higher binding energies, by $780 \mathrm{meV}$ to $284.95 \mathrm{eV}$. We conclude that the $\mathrm{Co}$ film is completely intercalated, since the structure of the $\mathrm{C} 1 \mathrm{~s}$ spectra reaches a stable shape with no further intensity changes or shifts in binding energy. The effective intercalation of a thin Co layer, and the formation of the graphene/Co/Ir(111), occurs at $450{ }^{\circ} \mathrm{C}$, identified by strong modifications of the $\mathrm{C}$ $1 s$ emission lines [Figs. 1(a) and 1(b)]. The $\mathrm{C} 1 s$ peak now has an additional second component at lower binding energies $(284.35 \mathrm{eV})$. This could be similar to the case of graphene on bulk Ni(111), where different absorption geometries of the carbon atoms (top-fcc and bridge-top) coexist, leading to a second component in the $\mathrm{C} 1 s$ spectrum at $284.46 \mathrm{eV}$ [27]. The second component emerges here upon cooling. We performed a separate experiment using $\mathrm{Ni}$ as intercalant, and assign this line, in analogy with the case of intercalated $\mathrm{Ni}$, to the different adsorption geometries of the carbon atoms. Furthermore, investigating the properties of the graphene/ $\mathrm{Ni} / \mathrm{Ir}(111)$ system, Pacile et al. [25] observe a strong asymmetry towards lower binding energy in the $\mathrm{C} 1 s$ line shape, which indicates the presence of a second component as well. The fact that the spectra presented in [25] were measured at room temperature with lower resolution, whereas the spectra in Fig. 1(a) were measured with high resolution at low temperature $(T=90$ $\mathrm{K})$, could account for the line shape with two clearly resolved components in the latter case. In the Ir $4 f$ spectra, the interface component is suppressed and only the two bulk components of the Ir $4 f$ levels remain.

The changes induced by intercalation of $\mathrm{Co}$ are even more strongly reflected in the valence electronic structure, as evident from the angle-resolved photoemission spectroscopy (ARPES) data in Fig. 2. In the top left dispersion plot, which shows the photoemission signal intensity along the high-symmetry directions of the graphene Brillouin zone, the sharp $\pi$ band, extending from about $8 \mathrm{eV}$ binding energy at the $\Gamma$ point right up to the Fermi energy at the $K$ point, i.e., the Brillouin-zone boundary, is clearly evident. The band exhibits small hybridization gaps where it collides with satellite $\pi$ bands brought about by the reciprocal lattice vectors of the large moiré structure unit cell, i.e., the lattice mismatch between graphene and $\operatorname{Ir}(111)$, as previously shown by Pletikosić et al. [29]. Sharp features in the region from 0 to $2-3 \mathrm{eV}$ binding energy are due to iridium bands of $d$ character, also visible in the constant energy cut on the right-hand side, at $0.3 \mathrm{eV}$ binding energy. The sharp features are assigned to Ir surface states (red), graphene Dirac cones (black) including the clearly visible satellite cones, and the bulk Ir bands, in very good agreement with literature data [30]. Intercalation [Fig. 2(b)] reduces the sharp features in the $d$-band region, or rather masks them through their dominating intensity, in the region from 0 to $2 \mathrm{eV}$ binding energy. Sharp dispersing features can still be seen here, e.g., between $\Gamma$ and $M$ and even more so between $K$ and $\Gamma$. The $\pi$ band, which is strongly shifted downwards (its bottom at $\Gamma$ shifted by more than 

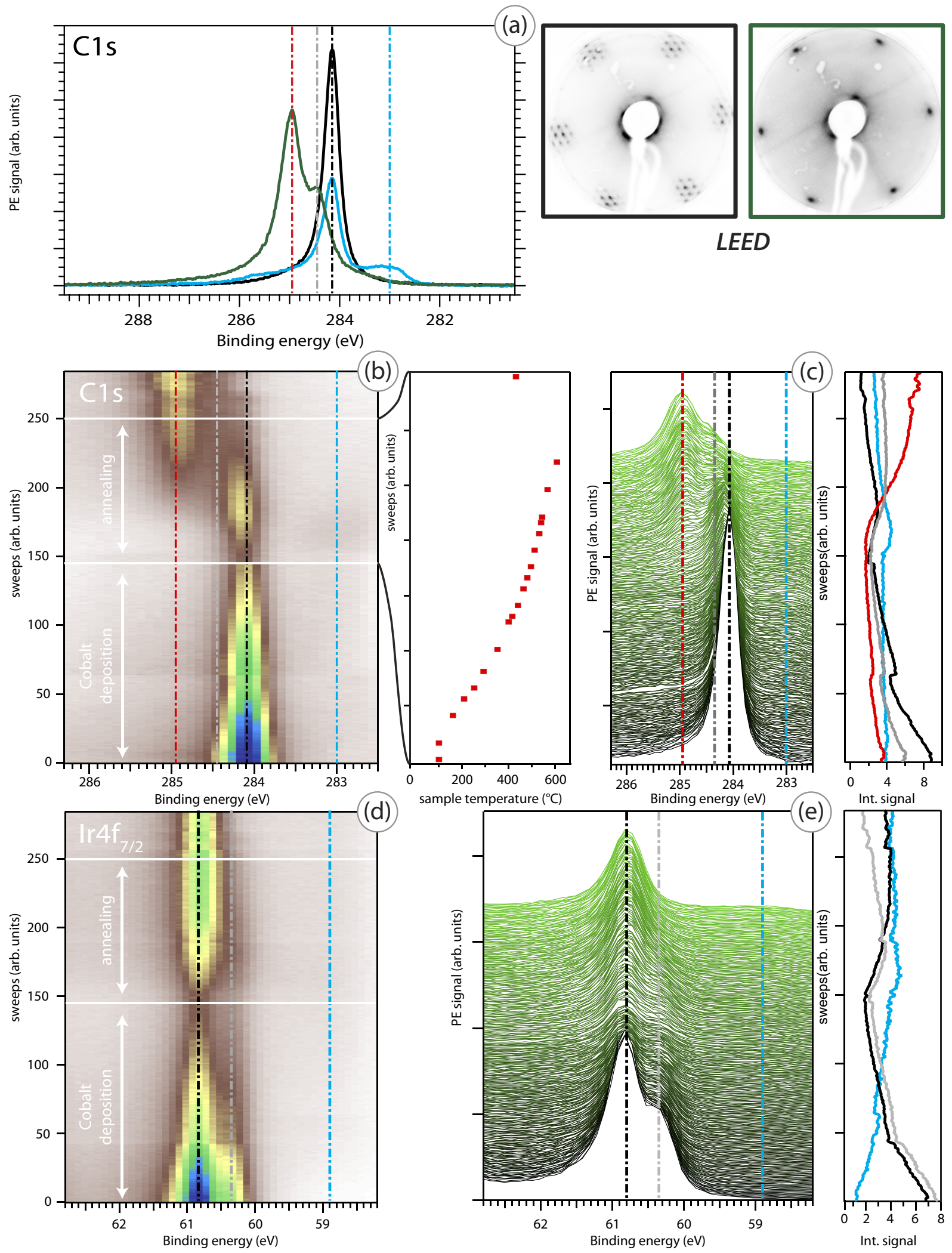

FIG. 1. (Color online) (a) Carbon C $1 s$ line spectra for pristine graphene/Ir(111) (black), Co on graphene/Ir(111) (blue), and intercalated graphene/Co/Ir(111) (green). (b),(d) Changes in C $1 s$ and Ir $4 f$ line shape upon deposition (up to sweep 145) and annealing, as false color plots [(b) and (d)] and waterfall plot [(c) and (e)]. Right side of (c) and (e): Extracted intensity at certain energies $E_{B}$ indicated by dashed lines in the core-level spectra, emphasizing the changes in the $\mathrm{C} 1 s$ and Ir $4 f$ line shape during intercalation. See text for discussion. 

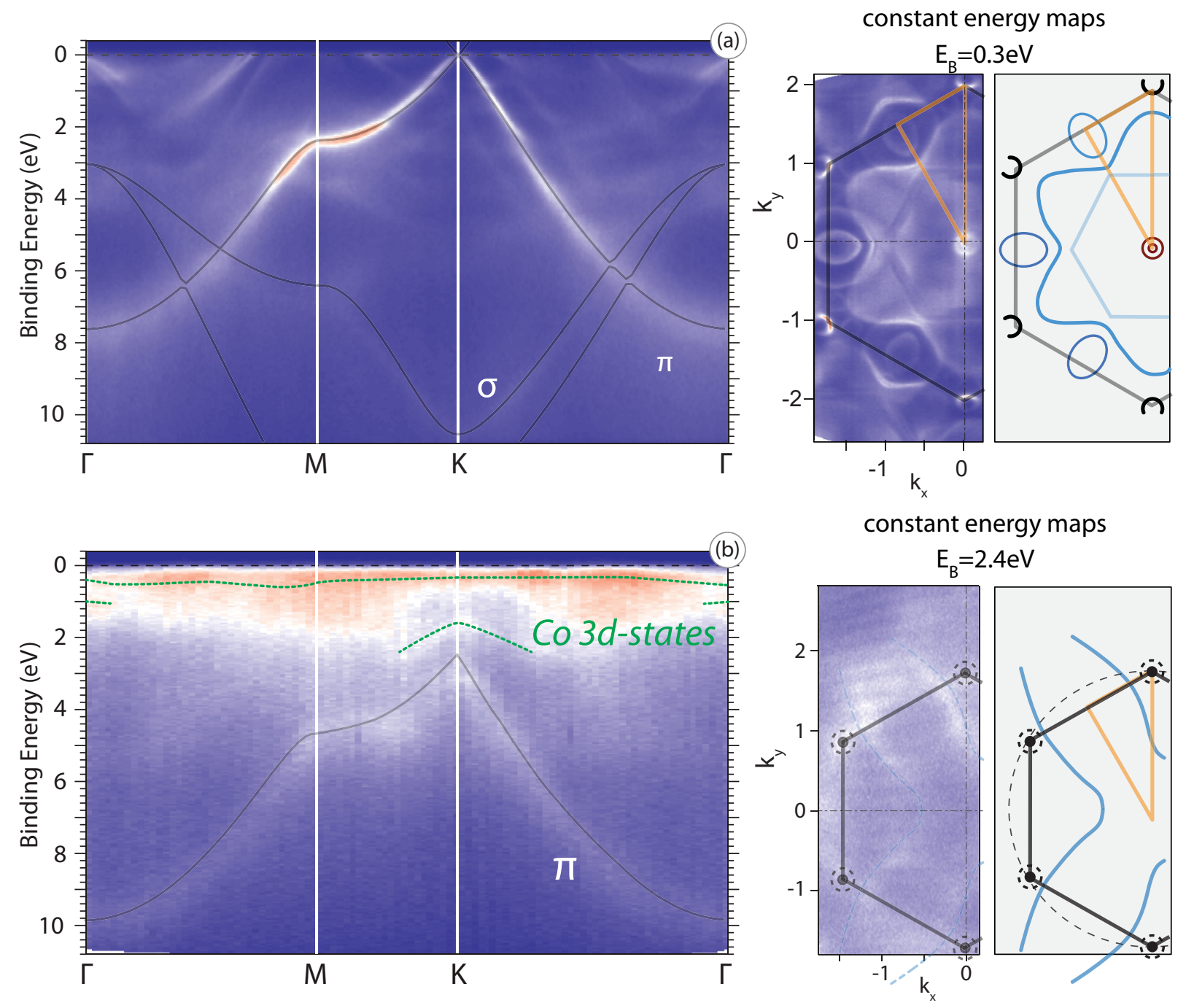

FIG. 2. (Color online) Electronic structure of graphene/Ir(111) (a) and intercalated graphene/Co/Ir (b) measured by ARPES. (a) Left side: ARPES intensity maps for the graphene layer on $\operatorname{Ir}(111)$ acquired along the $\Gamma-M-K-\Gamma$ direction of the BZ of graphene with photon energy $h v=65 \mathrm{eV}$. The grey line shows a simulated band structure for a free-standing graphene film [28]. Right side: constant energy surface at $E_{B}=0.3 \mathrm{eV}$ extracted from the ARPES measurements. Main features in red are derived from the $\operatorname{Ir}(111)$ surface state, graphene Dirac cones (black), and Ir bulk bands (blue). (b) Left side: Upon intercalation of Co, the graphene $\pi$ band is shifted to higher binding energy due to strong hybridization effects with Co $3 d$ states (photon energy $h v=94 \mathrm{eV}$ ). Main features of the Co film intercalated under graphene/Ir(111) are derived from Co $3 d$ states (green line) near the Fermi energy. Right side: constant energy surface at $E_{B}=2.4 \mathrm{eV}$ extracted from the ARPES measurements. Sketch of the constant energy surface shows the main features from trigonal shaped Ir $d$ states (blue line) of the substrate.

$2 \mathrm{eV}$ ), is weaker but can still be followed. It is also clear that the $\pi$ bands at $K$ end at about $2.2 \mathrm{eV}$, similar to previously published results for graphene/Co(0001) [31], albeit with a different interpretation.

In the constant energy plot of Fig. 2(b), clear evidence for dispersing Co $3 d$ bands is found; this supports our assumption that the Co intercalated layers are well ordered, and assume the lattice symmetry and site of the underlying Ir lattice.

Let us now turn to the main topic of this paper, i.e., the quantitative investigation of the magnetic properties of the intercalated Co layer for different thicknesses, and a comparison of these data with our theoretical predictions. To investigate the magnetic coupling behavior and to obtain quantitatively the related magnetic moments of the graphene/Co/Ir(111) system, we use x-ray magnetic circular dichroism (XMCD). First, our XMCD data for a single intercalated Co layer give no magnetic contrast at the Co $L_{2,3}$ edge. This is most likely due to insufficient sample cooling, since the Curie temperature for a single layer is expected to be below our sample temperature of $90 \mathrm{~K}$, in analogy with data from thin Ni films [32].

Increasing the amount of intercalated $\mathrm{Co}$, either in a single deposition/annealing cycle or several cycles, leads to the appearance of magnetic contrast as shown in Fig. 3. Quantitative data on the magnetic moments of the cobalt and carbon layers were evaluated from the absorption data as follows. The upper part of Fig. 3(a) shows the XAS intensity at the Co $L_{2,3}$ edge taken with different, right and left, circularly polarized light. The XMCD spectrum [black line in lower part of Fig. 3(a)] is obtained by the difference $\Delta I=I^{+}-I^{-}$, leading to a negative signal at the Co $L_{3}$ edge. The spectra are 

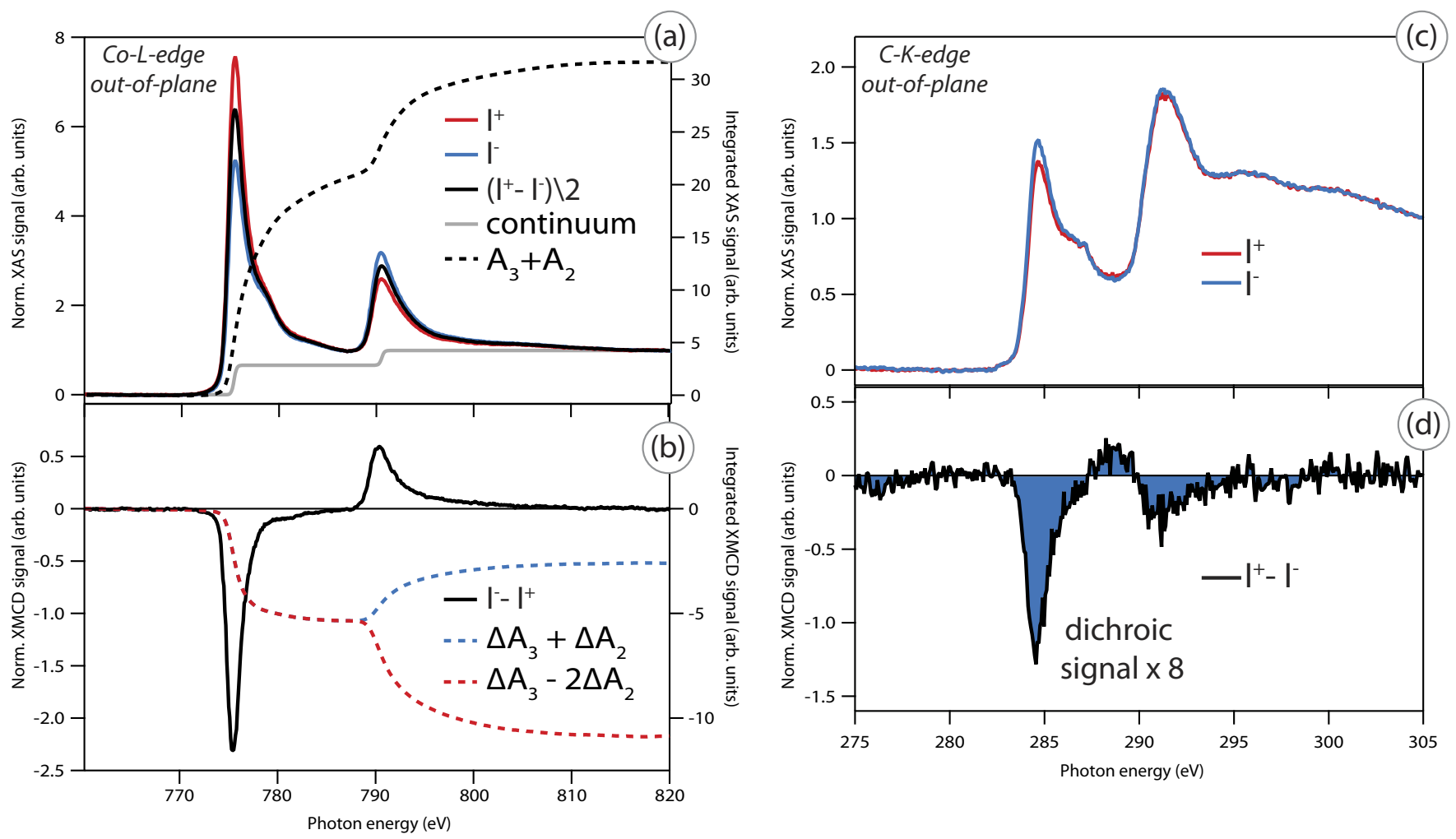

FIG. 3. (Color online) (a) XMCD spectra of the graphene/2 ML Co/Ir(111) system measured in an out-of-plane geometry at the Co $L_{2,3}$ absorption edge. The panels show the absorption spectra measured with circularly polarized light for two opposite directions (red and blue). The black line in (b) presents the corresponding difference spectra. The corresponding areas under the curves are indicated by broken lines. (c) XMCD spectra of the graphene/2-ML Co/Ir(111) system measured at the $\mathrm{C} K$ absorption edge. The panels show the absorption spectra measured with the circularly polarized light for two opposite directions (blue and red). The black line in (d) represents the corresponding difference spectra. The $\mathrm{C} K$ edge shows antiferromagnetic coupling compared to the Co $L_{2,3}$ absorption edge.

normalized to the edge jump by subtraction of a Fermi function taken at the center of the $L_{3}$ and $L_{2}$ edges, indicated as grey line in Fig. 3(a) [33]. For a quantitative evaluation we need the integrated intensities $A_{3}$ and $A_{2}$ under the $L_{3}$ and $L_{2}$ curves. The integrated intensity $A_{3}+A_{2}$ is formed by integrating the averaged XAS intensity $\frac{\left(I^{+}-I^{-}\right)}{2}$, which is also called "white line" [black curve in Fig. 3(a)] over the whole range of photon energy in the spectrum. The indices 2,3 indicate the ranges of photon energy of the Co $L_{2,3}$ edge. The integrals $\Delta A_{3}+\Delta A_{2}$ and $\Delta A_{3}-2 \Delta A_{2}$, which are needed for the evaluation of the magnetic moments below, are formed by integrating the XMCD signal over the range of photon energy in the spectrum.

To obtain quantitative values out of the XMCD spectra the following sum rules [34,35] are used:

$$
M_{L}=-\frac{2}{3} \frac{n_{h} \mu_{B}}{P_{\text {circ }} \cos \phi} \frac{\Delta A_{3}+\Delta A_{2}}{A_{3}+A_{2}}
$$

and

$$
M_{S}=-\frac{n_{h} \mu_{B}}{P_{\text {circ }} \cos \phi} \frac{\Delta A_{3}-2 \Delta A_{2}}{A_{3}+A_{2}}
$$

with $P_{\text {circ }}$ the degree of circular polarization of the incoming photons. The angle $\phi$ is defined by the geometry of the experiment, being the direction between the photon incidence and the magnetic moment. Since the magnetization of the sample and the $\vec{k}$ vector of the incident photons are collinear for out-of-plane measurements, the factor $\cos \phi$ yields unity. The number of holes in the Co $d$ band is given by $n_{h}$. From these sum rules, the ratio of the orbital and spin moment can be derived, which does not require magnetic saturation of the sample, which is useful for our measurements under reversal of the direction of the magnetic field.

Employing these XMCD sum rules, we extract the following numbers for the magnetic moments, using a number of $3 d$ holes $n_{h}=2.9$, according to the theory data presented below, and a degree of polarization of the light $P_{\text {circ }}=$ $75 \%$. At a sample temperature of $T=90 \mathrm{~K}$ our $2-\mathrm{ML}$ film of Co intercalated between graphene and $\operatorname{Ir}(111)$ exhibits an orbital moment of $M_{L}=0.15 \mu_{B}$ and a spin moment of $M_{S}=1.35 \mu_{B}$. We relate our results to the values for saturated magnetic moments of bulk Co $M_{L}=0.153 \mu_{B}$ and a spin moment of $M_{S}=1.55 \mu_{B}$ [33], which gives a ratio of $\frac{M_{L}}{M_{S}}=0.1$ for bulk Co. With $\frac{M_{L}}{M_{S}}=0.12$ we obtain slightly larger values for the graphene/Co/Ir(111) system. While the determination of $M_{S}$ and $M_{L}$ may be affected by the fact that full magnetic saturation was not achieved under our experimental conditions, the determination of this ratio does not suffer from such problems. The ratio is thus also significant for a comparison between theory and experiment.

An interesting finding is observed on the $\mathrm{C} K$ edge [Fig. 3(b)]: the C $K$ edge shows a fairly large dichroism in the leading $\pi^{\star}$ states around $285 \mathrm{eV}$ photon energy. The evidently large magnetic moment of the intercalated cobalt film 
is partially transferred onto the graphene states, a prerequisite for spin filtering [2].

To quantitatively interpret these findings one has to consider that there is a transition from a non-spin-orbit split $1 s$ initial state to $2 p$ final states. The analysis of the XMCD data at the C $K$ edge provides only information about the orbital magnetic moment. Following the description of Huang et al. [36] and Thole et al. [34] one can formulate a sum rule for $K$-edge absorption and relate this to orbital magnetic moments deduced by the $\mathrm{C} K$-edge dichroism signal :

$$
M_{L}=-\frac{1}{3} \frac{n_{h} \mu_{B}}{P_{\text {circ }} \cos \phi} \frac{\Delta A}{A} .
$$

For the C $K$ edge of the graphene/Co/Ir(111), measured under an angle of $\phi=40^{\circ}$, we find a fairly large dichroism signal [Fig. 3(d)]. We derive from the above formula a value of $M_{L}=0.1 \mu_{B}$. Studies on carbon nanotubes in contact with a flat ferromagnetic Co substrate yield similar results with a spin moment transfer of $0.1 \mu_{B}$ [37]. Furthermore, this value compares well to the determined numbers of induced magnetic moments in the graphene film by underlying ferromagnetic bulk material for the graphene/Ni(111)/W(110) system [2] and graphene/Fe/Ni(111) systems [4]. For graphene $/ \mathrm{Ni}(111) / \mathrm{W}(110)$ a magnetic moment in the range $M_{L}=0.05-0.1 \mu_{B}$ [3] was estimated, whereas in the graphene/Fe/Ni(111) system an increase in magnetic moment, compared to the latter case, by a factor of $\sim 2.7$ was observed [4]. From our measurements on graphene/Co/Ir(111) we find an increase in the magnetic moment by a factor of $\sim 2.0$ compared to graphene/Ni(111)/W(110). Thus we conclude that for the graphene/Co/Ir(111) system the strength of the induced magnetic moments at the $\mathrm{C} K$ edge lies in between the graphene/ $\mathrm{Ni}(111) / \mathrm{W}(110)$ and graphene/Fe/ $\mathrm{Ni}(111)$ cases. The dichroism occurs mostly on the $\pi^{\star}$ absorption edge, with a much smaller effect on the $\sigma^{\star}$ one. It cannot be excluded that the latter is caused by the background subtraction method.

Our data show an out-of-plane antiferromagetic coupling between the intercalated Co thin film and the graphene overlayer (Fig. 3), from the sign of the magnetic contrast in XMCD. These findings agree well with the previously published results [23], where the authors claim that the proposed magnetic moiré pattern has an overall antiferromagnetic ordering. Just as a reversal of the direction of the circular polarization of the light, reversing the magnetization direction should also induce magnetic circular dichroism. In the present case, a complete reversal of the direction of magnetization could not be achieved, most likely due to the insufficient strength of the magnet used in our study. However, the effect showed the correct trend, yielding similar results for the ratio $\frac{M_{L}}{M_{S}}$.

An intercalated cobalt layer presents an ideal situation to study emerging magnetism in ultrathin films, since unlike in deposited films, the morphology is expected to assume 2D layer growth, whereas deposited films tend to lead to clustering (Volmer-Weber growth). To understand the magnetic coupling in the intercalated cobalt thin film, related experiments using spin-polarized scanning tunneling microscopy (SP-STM) [23] and spin-polarized low-energy electron microscopy (SPLEEM) [22] were carried out by other groups. In SP-STM experiments it was observed that the intercalated cobalt film induces a magnetic moiré pattern in the graphene sheet, leading to ferromagnetic coupling between graphene and the underlying cobalt thin film at the atop sites and to antiferromagnetic coupling at the hcp/fcc sites; however, as mentioned above, the overall orientation was found to be antiferromagnetic. SPLEEM measurements showed that the graphene film induces a perpendicular magnetic anisotropy (PMA) in the underlying Co film. However, with both techniques it is not possible to extract quantitative information about the absolute size of the magnetic coupling. Utilizing XMCD at the Co $L_{2,3}$ and $\mathrm{C} K$ edges we gain quantitative insight into the magnetic behavior of the complex graphene/Co/Ir(111) system.

\section{ELECTRONIC STRUCTURE CALCULATIONS}

\section{A. Monolayer $\mathrm{Co} / \mathrm{Ir}(111)$ and $\mathrm{Graphene} / \mathrm{Co} / \mathrm{Ir}(111)$}

As discussed above, graphene forms a moiré superstructure on $\operatorname{Ir}(111)$ due to the in-plane lattice mismatch. In order to directly model this superstructure, one would need to consider $10 \times 10$ graphene unit cells (with $200 \mathrm{C}$ atoms) placed on a $9 \times 9 \operatorname{Ir}(111)$ mesh (with $81 \mathrm{Ir}$ atoms in each Ir layer), and insert a $9 \times 9$ Co layer between the graphene and the $\operatorname{Ir}(111)$ substrate. Application of density functional theory to such superstructure is a difficult task, taking into account that high accuracy relativistic calculations with spin-orbit coupling included are necessary for the analysis of the XMCD experimental results.

Instead of considering this large superstructure, we restrict ourselves to a more manageable system by placing two $\mathrm{C}$ atoms of the graphene unit cell on the top of a monolayer (ML) Co/Ir(111) [GR/[1ML Co]/Ir(111)], and consider three different placements for the graphene overlayer: top ("1-2"): one of the $\mathrm{C}$ atoms is on top of $\mathrm{Co}$, another is over an Ir atom of the interface ML; top ("1-3"): one of the C atoms is on top of $\mathrm{Co}$, another is over an Ir of the subinterface ML; hexagonal hollow ("2-3")" one of the C atoms is on top of the Ir interface $\mathrm{ML}$, another one is over the Ir subinterface ML. This supercell model, which consists of a ten-layer $\operatorname{Ir}(111)$ substrate and $1 \mathrm{ML}$ of Co on each side of the substrate, covered by a layer of graphene, is shown in Fig. 4. In addition, the properties of $1 \mathrm{ML}$ of Co on the $\operatorname{Ir}(111)$ surface [1 ML Co]/Ir(111) are calculated in order to analyze the graphene-induced changes in the magnetic properties of the Co atoms. A similar approach is used by Busse et al. [38] and Voloshina et al. [39], to describe the graphene/Ir(111) system compared to graphene/ $\mathrm{Ni}(111)$, utilizing hybrid states which allow the transfer of magnetic moments.

The structural optimization procedure is performed using the standard VASP-PAW [40] program package without spinorbit coupling (SOC) employing the generalized gradient approximation (GGA)-Perdew-Burke-Ernzerhof (PBE). The in-plane interatomic distance of pure Ir, 5.132 a.u. was adopted and kept fixed in the calculations. For [1-ML Co]/Ir(111), we obtain a relatively large $-8.4 \%$ relaxation of the interlayer distance $d_{[\mathrm{Co}-\mathrm{Ir}]}=3.84$ a.u. The $2.5 \%$ change in the distance between the Ir-interface (Ir-I) and the Ir-subinterface (Ir-I-1) layers $d_{[(\mathrm{Ir}-\mathrm{I})-(\mathrm{Ir}-\mathrm{I}-1)]}=4.31$ a.u. is substantial. Very smallpractically negligible - changes in the Ir atom positions for the rest of the substrate are found in the calculations. For 

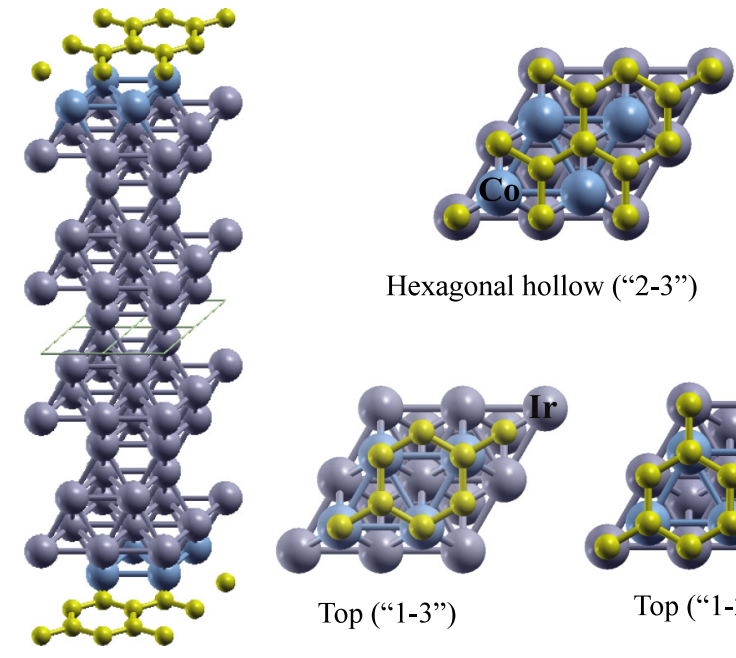

Hexagonal hollow ("2-3")

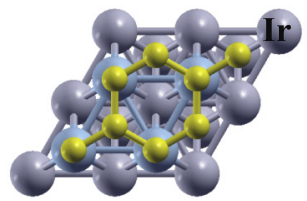

Top (“1-3”)

FIG. 4. (Color online) A schematic crystal structure used to represent the graphene/Co/Ir(111) surface.

GR/[1-ML Co]/Ir(111), in the cases of top (1-2) and top (1-3), we find that graphene is strongly bound to the Co atom, with $d_{[\mathrm{C}-\mathrm{Co}]}=3.80$ and 3.72 a.u. respectively. For the hexagonal hollow (2-3) case, the $\mathrm{C}$ atoms are much less connected to the substrate, with $d_{[\mathrm{C}-\mathrm{Co}]}=4.27$ a.u.

Once the structure relaxation is carried out, we use the relativistic version of the full-potential linearized augmented plane-wave method (FP-LAPW) [41], in which SOC is included in a self-consistent second-variational procedure [42], and the local-spin-density (LSDA)-von Barth-Hedin approximation is adopted. The radii of the atomic muffin-tin (MT) spheres are set to 1.4 a.u. for $\mathrm{C}$ atoms, 2.2 a.u. for $\mathrm{Co}$, and 2.5 a.u. for Ir atoms. The parameter $R_{\mathrm{Co}} \times K_{\max }=7.7$ defines the basis set size, and the Brillouin zone was sampled with $229 k$ points. In all calculations the magnetization is directed along the $z$ axis along the surface normal. The use of the relativistic FP-LAPW method allows an accurate determination of the element-specific spin $\left(M_{S}\right)$ and orbital $\left(M_{L}\right)$ magnetic moments.

Spin $\left(M_{S}\right)$ and orbital $\left(M_{L}\right)$ magnetic muffin-tin moments are shown in Table I for the Co, Ir interface (I), subinterface (I-1), and subsubinterface (I-2) layers. There is a reduction of the $M_{S}$ and $M_{L}$ values of the Co layer as compared to the unrelaxed case $\left(M_{S}=1.89 \mu_{B}, M_{L}=0.13 \mu_{B}\right)$, and an enhancement of the magnetic polarization of the substrate. This is consistent with the structure relaxation-induced reduction of the Co-Ir distance $d_{[\text {Co-Ir] }}$, leading to an increase of hybridization between the Co overlayer and the Ir substrate. The Ir interfacial layer is spin polarized parallel to the Co ML, while the spin-polarisation for the Ir-(I-1) and Ir-(I-2) layers is antiparallel. The spin moments are practically zero for the $\mathrm{C}$ atoms of the graphene ML in case of the 2-3 position, reflecting a very weak interaction between the Co-ML and graphene. Once the $\mathrm{C}$ atom interacts with the Co atom underneath, as in the case of 1-2 and 1-3, the spin magnetic moments are induced. The total induced spin moment in graphene is very small ( $\approx 0.01 \mu_{B}$ per unit cell of graphene), and it is interesting to note that it is oriented antiparallel to the Co-ML moment.
TABLE I. Spin $\left(M_{S}\right)$ and orbital $\left(M_{L}\right)$ magnetic moments in the MT sphere of the $\mathrm{C}, \mathrm{Co}$, and $\mathrm{Ir}$ atoms (in Bohr magnetons) for [1-ML Co]/Ir(111) and GR/[1-ML Co]/Ir(111).

\begin{tabular}{|c|c|c|c|c|c|c|}
\hline \multicolumn{7}{|c|}{$[1 \mathrm{ML} \mathrm{Co}] / \operatorname{Ir}(111)$} \\
\hline & & & Co & Ir $[\mathrm{I}]$ & $\operatorname{Ir}[\mathrm{I}-1]$ & $\operatorname{Ir}[\mathrm{I}-2]$ \\
\hline$M_{S}$ & & & 1.80 & 0.21 & -0.06 & -0.02 \\
\hline$M_{L}$ & & & 0.12 & 0.01 & -0.00 & -0.00 \\
\hline \multicolumn{7}{|c|}{ GR/[1ML Co]/Ir(111) } \\
\hline \multicolumn{7}{|c|}{ Hexagonal hollow 2-3 } \\
\hline & $\mathrm{C}(1)$ & $\mathrm{C}(2)$ & Co & $\operatorname{Ir}(\mathrm{I})$ & $\operatorname{Ir}(\mathrm{I}-1)$ & $\operatorname{Ir}(\mathrm{I}-2)$ \\
\hline$M_{S}$ & 0.00 & 0.00 & 1.57 & 0.19 & -0.06 & -0.02 \\
\hline$M_{L}$ & 0.00 & 0.00 & 0.08 & 0.00 & -0.00 & 0.00 \\
\hline \multicolumn{7}{|c|}{ Top 1-3 } \\
\hline & $\mathrm{C}(1)$ & $\mathrm{C}(2)$ & Co & $\operatorname{Ir}(\mathrm{I})$ & $\operatorname{Ir}(\mathrm{I}-1)$ & $\operatorname{Ir}(\mathrm{I}-2)$ \\
\hline$M_{S}$ & -0.02 & 0.02 & 1.16 & 0.10 & -0.05 & -0.02 \\
\hline$M_{L}$ & 0.00 & 0.00 & 0.07 & 0.00 & -0.01 & 0.00 \\
\hline \multicolumn{7}{|c|}{ Top 1-2 } \\
\hline & $\mathrm{C}(1)$ & $\mathrm{C}(2)$ & Co & $\operatorname{Ir}(\mathrm{I})$ & $\operatorname{Ir}(\mathrm{I}-1)$ & $\operatorname{Ir}(\mathrm{I}-2)$ \\
\hline$M_{S}$ & -0.03 & 0.02 & 1.29 & 0.13 & -0.04 & -0.02 \\
\hline$M_{L}$ & 0.00 & 0.00 & 0.07 & 0.00 & 0.00 & 0.00 \\
\hline
\end{tabular}

Note that similar spin magnetic moments at the $\mathrm{C}$ atoms are reported for graphene/ $\mathrm{Ni}(111)$ [43].

Let us compare our data with previously reported graphene/Co/Ir(111) calculations by Decker et al. [23]. They reported DFT $+U$ calculations of a supercell which contained $(10 \times 10)$ graphene unit cells placed on a $(9 \times 9)$ monolayer of Co and three layers of the $\operatorname{Ir}(111)$ substrate. The values of spin-only moments on the Co atoms were presented. No value for the orbital magnetic moments on the Co and Ir atoms were given [44], instead, they reported a spin moment of $-1.36 \mu_{B}$ for two $\mathrm{C}$ atoms in a graphene unit cell in the 1-3 and 1-2 positions ("fcc" and "hcp" in their notations), coupled antiparallel to the Co layer beneath. For the 2-3 case, a graphene unit-cell spin moment of $0.14 \mu_{B}$ coupled parallel to the Co layer was reported. We do not find such strong spin polarization in graphene on top of the Co layer (see Table I), at least in the muffin-tin spheres around the $\mathrm{C}$ atoms, but the orientations of the graphene moments are qualitatively consistent with those reported in Ref. [23].

\section{B. Graphene/[2-ML Co]//r(111)}

Now we turn to a salient aspect of our investigation, a comparison between the XMCD experiments and our calculations for two monolayers of Co intercalated in between graphene and $\operatorname{Ir}(111)$. We employ the same supercell approach as described above (see Fig. 4) inserting an extra Co-ML into GR/[1-ML Co]/Ir(111). Again we make use of a two-step procedure: at first, the relaxed structure is obtained with the VASP code without the SOC; next, the FP-LAPW calculations including SOC are performed. Since we noticed for the case of GR/[1-ML Co]/Ir(111) that for the 1-3 and 1-2 placements of graphene, there are only minor differences for the Co atom magnetic moments, only the 2-3 and 1-3 cases are considered.

We show in Table II the spin $M_{S}$, orbital $M_{L}$, and magnetic dipole moment $M_{D}$ (in $\mu_{B}$ ) (note that in our notations, 
TABLE II. Spin $\left(M_{S}\right)$, orbital $\left(M_{L}\right)$, and dipole $\left(M_{D}\right)$ magnetic moments (in Bohr magnetons) in the $d$ shells, and the ratio $R_{L S}=$ $\frac{M_{L}}{M_{S}+M_{D}}$ for a double layer of Co intercalated in between graphene and $\operatorname{Ir}(111)$ : for the Co monolayer next to the graphene (Co@GR) and the Co monolayer next to the Ir substrate (Co@ Ir) for different graphene overlayer placements.

\begin{tabular}{lcrrrr}
\hline \hline & \multicolumn{3}{c}{ Co@GR } & \multicolumn{2}{c}{ Co@Ir } \\
& & \multicolumn{1}{c}{$2-3$} & \multicolumn{1}{c}{$1-3$} & \multicolumn{1}{c}{$2-3$} & \multicolumn{1}{c}{$1-3$} \\
\hline \multirow{3}{*}{ LSDA } & $M_{S}$ & 1.52 & 1.56 & 1.69 & 1.66 \\
& $M_{L}$ & 0.07 & 0.09 & 0.09 & 0.09 \\
& $M_{D}$ & -0.31 & -0.01 & -0.08 & -0.07 \\
& $R_{L S}$ & 0.06 & 0.06 & 0.06 & 0.06 \\
\hline \multirow{3}{*}{ LSDA+U-FLL } & $M_{S}$ & 1.73 & 1.72 & 1.92 & 1.90 \\
& $M_{L}$ & 0.20 & 0.24 & 0.28 & 0.27 \\
& $M_{D}$ & -0.47 & -0.10 & -0.11 & -0.13 \\
& $R_{L S}$ & 0.16 & 0.15 & 0.16 & 0.16 \\
\hline \multirow{3}{*}{ LSDA+U-AMF } & $M_{S}$ & 1.52 & 1.48 & 1.66 & 1.62 \\
& $M_{L}$ & 0.13 & 0.19 & 0.19 & 0.18 \\
& $M_{D}$ & -0.32 & 0.13 & 0.014 & -0.01 \\
& $R_{L S}$ & 0.11 & 0.11 & 0.11 & 0.12 \\
\hline \hline
\end{tabular}

$M_{D}=7\left\langle T_{z}\right\rangle$, where the $\left\langle T_{z}\right\rangle=\frac{1}{7} Q_{z z} M_{S}[45,46]$, and $Q_{z z}$ is a quadrupole moment), and the ratio $R_{L S}$ for the $d$ shell of the Co monolayer next to graphene (Co@GR) and the Co monolayer next to the Ir substrate (Co@Ir). For the hexagonal hollow 2-3 position of the graphene overlayer, the LSDA calculations yield for Co@GR a $d$-shell occupation of 7.14, and for the 1-3 position $n_{d}=7.10$, while for Co@ $\mathrm{Ir}, n_{d}=7.03-7.04$.

We find that the $M_{S}, M_{L}$, and $M_{D}$ moments depend on the graphene overlayer placement as well as on the position of the Co atom (Co@GR, Co@ Ir). These differences are small except for the Co@GR case where both $M_{L}$ and $M_{D}$ are changing. This can be traced to the changes in the electronic structure due to the charge redistribution in the Co atom $d$ shell. Namely, with a change in the graphene overlayer placement from 1-3 to 2-3 (see Fig. 4), the occupation for the spin-minority $\left\{3 z^{2}-r^{2}\right\}$ orbital increases, and $Q_{z z}$ becomes more negative so that the sum $\left[M_{S}+M_{D}\right]$ is reduced. Simultaneously, the orbital magnetic moment $M_{L}$ is reduced. Thus, the ratio $R_{L S}$ is less affected by the graphene overlayer placement, and remains close to 0.06 , i.e., half the value which is measured by the XMCD experiment.

It is well known that LSDA does not account properly for the orbital polarization in the transitional $d$ metals, and underestimates the values of the orbital magnetic moments [47]. In order to analyze this effect, we have applied the rotationally invariant LSDA $+U$ method, which preserves the full local occupation matrix including all spin off-diagonal components. Two flavors of LDA $+U$ with different choices for the double-counting term, the "fully localized" (FLL) [48] and the "around-mean-field" (AMF) [49], were considered. A Coulomb $U$ of $3 \mathrm{eV}$ was chosen, from an average of the $U$ values commonly used in the LDA $+U$ calculations of the transitional metals [50]. An exchange $J$ of $0.9 \mathrm{eV}$ was used which corresponds to a choice of the Slater integrals of $F_{2}=7.75 \mathrm{eV}$, and $F_{4}=4.85 \mathrm{eV}$.

The spin $M_{S}$, orbital $M_{L}$, and magnetic dipole moment $M_{D}$ (in $\mu_{B}$ ), and the ratio $R_{L S}$ calculated with LSDA $+U$ -
FLL are also shown in Table II. We find that the spin and orbital magnetic moments for both Co@GR and Co@Ir layers are enhanced over the LSDA values. The magnetic dipole moments also increase in magnitude. The $d$-shell occupation is $n_{d}=7.12$ (Co@GR, 1-3 and 2-3), and $n_{d}=7.03-7.04$ for (Co@Ir, 1-3 and 2-3). Similar to LSDA, the ratio $R_{L S}$ is about $0.15-0.16$, closer to but now exceeding the experimental XMCD value of 0.12 . Note that for the [1-ML Co]/Ir(111) case calculated with LSDA $+U$-FLL and the same values of the Coulomb $U$ and exchange $J$, the values are $n_{d}=7.02$, $M_{S}=2.07 \mu_{B}, M_{L}=0.493 \mu_{B}$, magnetic dipole moment, $M_{D}=0.32 \mu_{B}$, and the ratio $R_{L S}$ is 0.21 .

Once the LSDA $+U$-AMF is applied, this ratio becomes $0.11-0.12$ in good agreement with the XMCD experiment. The $d$-shell occupation remains practically unchanged, $n_{d}=$ 7.11-7.14 (Co@GR, 1-3 and 2-3), and $n_{d}=7.03-7.04$ for (Co@Ir, 1-3 and 2-3). The values of individual moments are
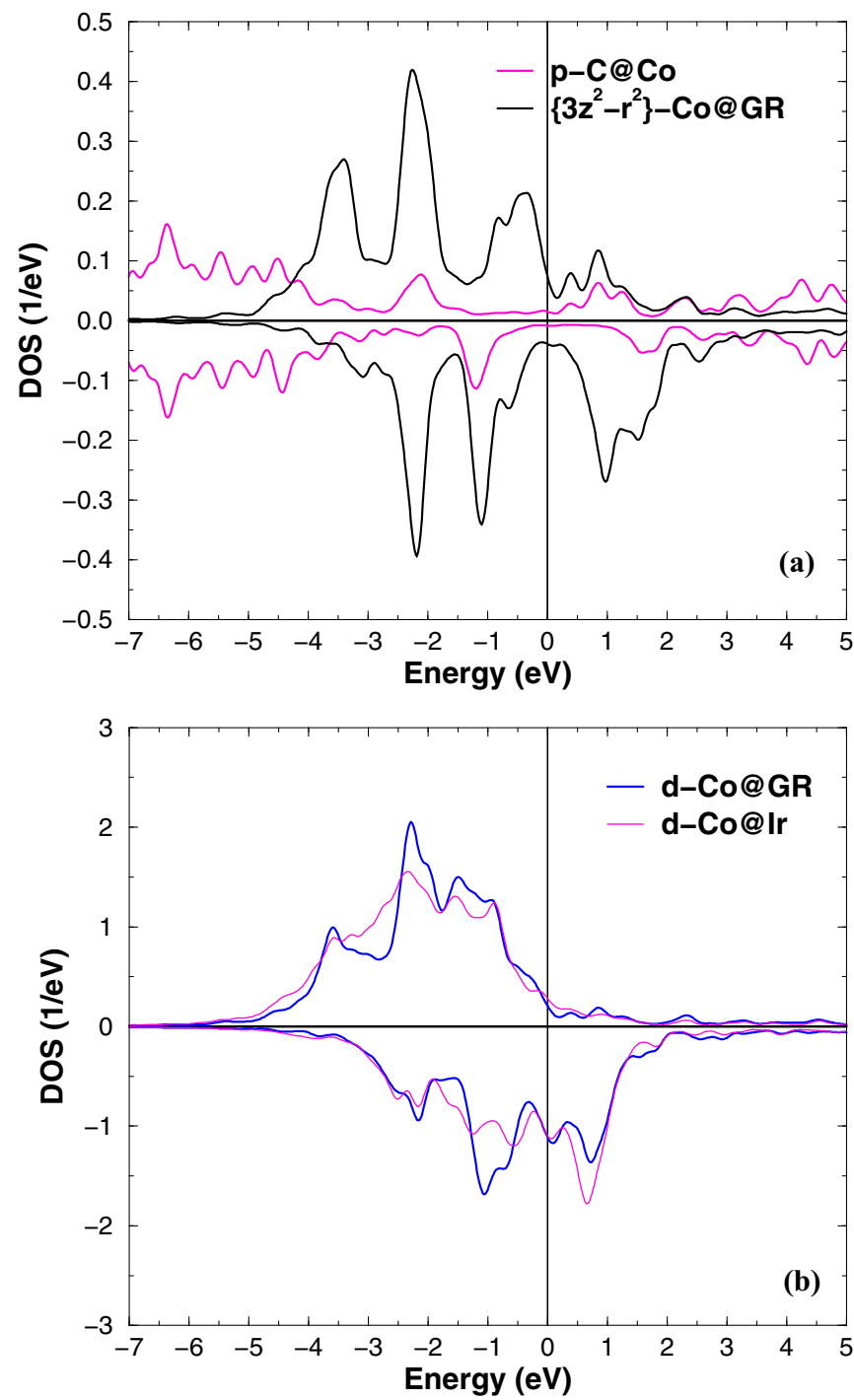

FIG. 5. (Color online) (a) Spin-resolved projected DOS for the $p$ states of $\mathrm{C}$ atom on top of $\mathrm{Co}$, together with the $\left\{3 z^{2}-r^{2}\right\}$ projected DOS for the $d$ states of Co@GR atom; (b) spin-resolved DOS for the $d$ states of Co@GR and Co@Ir in top (1-3) case calculated with relativistic $\mathrm{LSDA}+U-\mathrm{AMF}(U=3 \mathrm{eV})$. 
listed in Table II. Again, it is seen that the $M_{S}, M_{L}$, and $M_{D}$ moments depend on the graphene overlayer placement as well as on the position of the Co atom (Co@GR, Co@ Ir). While both the sum $\left[M_{S}+M_{D}\right]$ and $M_{L}$ are changing, the ratio $R_{L S}$ remains unaffected. For a clean [1-ML Co]/Ir(111) calculated with LSDA $+U$-AMF and the same values of the Coulomb $U$ and exchange $J$, we get a $d$-shell occupation of 7.03, $M_{S}=1.78 \mu_{B}, M_{L}=0.29 \mu_{B}$, magnetic dipole moment, $M_{D}=0.29 \mu_{B}$, and a ratio $R_{L S}=0.140$.

For the 2-3 case, small spin moments $M_{S}$ are induced on the $\mathrm{C}$ atoms $\left(-0.012 \mu_{B}, 0.001 \mu_{B}\right)$. For the 1-3 case, the moments are larger, with $M_{S}=-0.027 \mu_{B}$ for the $\mathrm{C}$ atom on the top of $\mathrm{Co}\left(\mathrm{C} @ \mathrm{Co}\right.$ ), and $M_{S}=0.014 \mu_{B}$ for the $\mathrm{C}$ atom over the Ir substrate (C@Ir). These moments are mainly of $p$-orbital character and originate from spin-dependent hybridization between $\mathrm{C} \pi$ and Co $d$ valence-band states, similar to the graphene/Ni(111) case [3]. The spin-resolved projected density of states (DOS) for the $p$ states of the $\mathrm{C}$ atom on the top of Co $(\mathrm{C} @ \mathrm{Co})$ is shown in Fig. 5. It is seen that the $\mathrm{C}$ atom spin polarization follows the spin polarization of the $\left\{3 z^{2}-r^{2}\right\} d$ states of the Co@GR atom. This spin splitting of the $p$ states is qualitatively consistent with the carbon $K$-edge XMCD spectra in Fig. 3(d). However, the theory does not support the sizable orbital moment $M_{L}$ of the $p$ states of the $\mathrm{C}$ atom. This moment is derived from the XMCD experimental data making use of the orbital moment sum rule [Eq. (3)]. The reason for this disagreement is not clear at the moment. It can indicate limitations of the DFT calculations for a proper description of orbital polarization in graphene, and the necessity to go beyond DFT for the $\mathrm{C}$ atoms of graphene. Another possibility is that the use of the commensurate in-plane unit cell of graphene instead of a realistic moiré pattern can lead to neglect of strong interface effects such as charge-transfer induced polarization in the adsorbed graphene. From the computational point of view accurate evaluation of a graphene orbital moment in a realistic moiré structure remains a challenge.

The spin-resolved DOS for the $d$ states of Co@GR and Co@Ir in the top (1-3) case, calculated with relativistic
LSDA $+U$-AMF $(U=3 \mathrm{eV})$ are shown in Fig. 5. It is seen that the spin splitting of the $d$ states is slightly reduced for Co@GR as compared to Co@Ir. This reduction is consistent with the corresponding reduction of the spin moment $M_{S}$ seen in Table II. We can interpret it in terms of spin-polarization transfer from the Co layer to graphene, mainly due to hybridization between $\mathrm{C} \pi$ and $\operatorname{Co}\left\{3 z^{2}-r^{2}\right\} d$ states.

\section{SUMMARY}

We characterize the growth and electronic as well as the magnetic structure of cobalt layers intercalated in between graphene on $\operatorname{Ir}(111)$ using a combination of photoemission and XMCD spectroscopy. It is demonstrated that magnetic ordering exists beyond monolayer intercalation. The orbital and spin magnetic moments of the Co atoms are quantitatively determined. The carbon edge XMCD shows an induced magnetic moment in the graphene layer, oriented antiparallel to that of cobalt. The XMCD experimental data are discussed in comparison to our results of first-principles electronic structure calculations. It is shown that good quantitative agreement between theory and experiment for the Co magnetic moments is achieved, when the local-spin-density approximation plus Hubbard $U(\operatorname{LSDA}+U)$ is used.

\section{ACKNOWLEDGMENTS}

The authors wish to thank A. Preobrajenski from MAX-lab, W. Mahler and B. Zada from BESSY-II for technical assistance and support during beamtime, and Y. S. Dedkov for useful discussion. This work has been supported by the European Science Foundation (ESF) under the EUROCORES Programme, EuroGRAPHENE, Project "SpinGraph", funded through the German Research Foundation (DFG) Project No. H0 797/18-1. H.V. appreciates the support from the German Research Foundation (DFG; Grant No. DE 1679/3-1) through the Priority Program (SPP) 1459 "Graphene." The support from Czech Republic GACR Grants No. P204/10/0330 and No. $14-37427 \mathrm{G}$ is acknowledged.
[1] Y. S. Dedkov, K. Horn, A. B. Preobrajenski, and M. Fonin, in Graphene Nanoelectronics, edited by H. Raza (Springer, Berlin-Heidelberg, 2012), pp. 189-234.

[2] Y. S. Dedkov and M. Fonin, New J. Phys. 12, 125004 (2010).

[3] M. Weser, Y. Rehder, K. Horn, M. Sicot, M. Fonin, A. B. Preobrajenski, E. N. Voloshina, E. Goering, and Y. S. Dedkov, Appl. Phys. Lett. 96, 012504 (2010).

[4] M. Weser, E. N. Voloshina, K. Horn, and Y. S. Dedkov, Phys. Chem. Chem. Phys. 13, 7534 (2011).

[5] V. M. Karpan, G. Giovannetti, P. A. Khomyakov, M. Talanana, A. A. Starikov, M. Zwierzycki, J. van den Brink, G. Brocks, and P. J. Kelly, Phys. Rev. Lett. 99, 176602 (2007).

[6] M. Gyamfi, T. Eelbo, M. Wasniowska, and R. Wiesendanger, Phys. Rev. B 85, 205434 (2012).

[7] H. Hattab, A. T. N'Diaye, D. Wall, G. Jnawali, J. Coraux, C. Busse, R. van Gastel, B. Poelsema, T. Michely, F. J. M. Z.
Heringdorf, and M. Horn-von Hoegen, Appl. Phys. Lett. 98, 141903 (2011).

[8] A. T. N'Diaye, J. Coraux, T. N. Plasa, C. Busse, and T. Michely, New J. Phys. 10, 043033 (2008).

[9] D. Farias, A. M. Shikin, K. H. Rieder, and Y. S. Dedkov, J. Phys.: Condens. Matter 11, 8453 (1999).

[10] Y. S. Dedkov, A. M. Shikin, V. K. Adamchuk, S. L. Molodtsov, C. Laubschat, A. Bauer, and G. Kaindl, Phys. Rev. B 64, 035405 (2001).

[11] A. Nagashima, N. Tejima, and C. Oshima, Phys. Rev. B 50, 17487 (1994).

[12] A. M. Shikin, D. Farias, and K. H. Rieder, Europhys. Lett. 44, 44 (1998).

[13] A. M. Shikin, G. V. Prudnikova, V. K. Adamchuk, F. Moresco, and K. H. Rieder, Phys. Rev. B 62, 13202 (2000).

[14] D. Farias, K. H. Rieder, A. M. Shikin, V. K. Adamchuk, T. Tanaka, and C. Oshima, Surf. Sci. 454, 437 (2000). 
[15] A. M. Shikin, Y. S. Dedkov, V. K. Adamchuk, D. Farias, and K. H. Rieder, Surf. Sci. 452, 1 (2000).

[16] A. M. Shikin, D. Farias, V. K. Adamchuk, and K. H. Rieder, Surf. Sci. 424, 155 (1999).

[17] A. Cupolillo, N. Ligato, and L. S. Caputi, Appl. Phys. Lett. 102, 111609 (2013).

[18] A. Politano and G. Chiarello, Nanoscale 6, 10927 (2014).

[19] E. N. Voloshina, A. Generalov, M. Weser, S. Bottcher, K. Horn, and Y. S. Dedkov, New J. Phys. 13, 113028 (2011).

[20] C. Riedl, C. Coletti, T. Iwasaki, A. A. Zakharov, and U. Starke, Phys. Rev. Lett. 103, 246804 (2009).

[21] J. Coraux, A. T. N'Diaye, N. Rougemaille, C. Vo-Van, A. Kimouche, H. X. Yang, M. Chshiev, N. Bendiab, O. Fruchart, and A. K. Schmid, J. Phys. Chem. Lett. 3, 2059 (2012).

[22] N. Rougemaille, A. T. N'Diaye, J. Coraux, C. Vo-Van, O. Fruchart, and A. K. Schmid, Appl. Phys. Lett. 101, 142403 (2012).

[23] R. Decker, J. Brede, N. Atodiresei, V. Caciuc, S. Blugel, and R. Wiesendanger, Phys. Rev. B 87, 041403(R) (2013).

[24] S. Valic, A. Kimouche, J. Coraux, B. Santos, A. Locatelli, and N. Rougemaille, Appl. Phys. Lett. 104, 101602 (2014).

[25] D. Pacile, P. Leicht, M. Papagno, P. M. Sheverdyaeva, P. Moras, C. Carbone, K. Krausert, L. Zielke, M. Fonin, Y. S. Dedkov, F. Mittendorfer, J. Doppler, A. Garhofer, and J. Redinger, Phys. Rev. B 87, 035420 (2013).

[26] D. X. Ye, S. Pimanpang, C. Jezewski, F. Tang, J. J. Senkevich, G. C. Wang, and T. M. Lu, Thin Solid Films 485, 95 (2005).

[27] W. Zhao, S. M. Kozlov, O. Hofert, K. Gotterbarm, M. P. A. Lorenz, F. Vines, C. Papp, A. Gorling, and H.-P. Steinruck, J. Phys. Chem. Lett. 2, 759 (2011).

[28] S. Boettcher, Master's thesis, Freie Universität, Berlin, 2010.

[29] I. Pletikosić, M. Kralj, P. Pervan, R. Brako, J. Coraux, Alpha T. N'Diaye, C. Busse, and T. Michely, Phys. Rev. Lett. 102, 056808 (2009).

[30] E. Starodub, A. Bostwick, L. Moreschini, S. Nie, F. E. Gabaly, K. F. McCarty, and E. Rotenberg, Phys. Rev. B 83, 125428 (2011).

[31] A. Varykhalov and O. Rader, Phys. Rev. B 80, 035437 (2009).

[32] K. Baberschke, Appl. Phys. A: Mater. Sci. Process. 62, 417 (1996).

[33] C. T. Chen, Y. U. Idzerda, H.-J. Lin, N. V. Smith, G. Meigs, E. Chaban, G. H. Ho, E. Pellegrin, and F. Sette, Phys. Rev. Lett. 75, 152 (1995).
[34] B. T. Thole, P. Carra, F. Sette, and G. van der Laan, Phys. Rev. Lett. 68, 1943 (1992).

[35] P. Carra, B. T. Thole, M. Altarelli, and X. Wang, Phys. Rev. Lett. 70, 694 (1993).

[36] D. J. Huang, H. T. Jeng, C. F. Chang, G. Y. Guo, J. Chen, W. P. Wu, S. C. Chung, S. G. Shyu, C. C. Wu, H. J. Lin, and C. T. Chen, Phys. Rev. B 66, 174440 (2002).

[37] O. Céspedes, M. S. Ferreira, S. Sanvito, M. Kociak, and J. M. D. Coey, J. Phys.: Condens. Matter 16, L155 (2004).

[38] C. Busse, P. Lazic, R. Djemour, J. Coraux, T. Gerber, N. Atodiresei, V. Caciuc, R. Brako, Alpha T. N'Diaye, S. Blugel, J. Zegenhagen, and T. Michely, Phys. Rev. Lett. 107, 036101 (2011).

[39] E. N. Voloshina, E. Fertitta, A. Garhofer, F. Mittendorfer, M. Fonin, A. Thissen, and Y. S. Dedkov, Sci. Rep. 3, 1072 (2013).

[40] G. Kresse and J. Hafner, Phys. Rev. B 47, 558 (1993); G. Kresse and J. Furthmuller, Comput. Mater. Sci. 6, 15 (1996); G. Kresse and D. Joubert, Phys. Rev. B 59, 1758 (1999).

[41] D. J. Singh, Planewaves, Pseudopotentials and the LAPW Method (Kluwer Academic, Boston, 1994), p. 115.

[42] A. B. Shick, D. L. Novikov, and A. J. Freeman, Phys. Rev. B 56, R14259 (1997).

[43] L. V. Dzemiantsova, M. Karolak, F. Lofink, A. Kubetzka, B. Sachs, K. von Bergmann, S. Hankemeier, T. O. Wehling, R. Fromter, H. P. Oepen, A. I. Lichtenstein, and R. Wiesendanger, Phys. Rev. B 84, 205431 (2011).

[44] The spin moments from DFT $+U$ [23] differ from the LSDA values listed in Table I. Once the LSDA $+U$ is used with $U=3 \mathrm{eV}$ and $J=0.9 \mathrm{eV}$, spin $M_{S}=1.91 \mu_{B}$ (for the 2-3 case), $M_{S}=1.67 \mu_{B}(1-2)$, and $M_{S}=1.47 \mu_{B}$ (1-3) are calculated. They are substantially close to the moments reported in Ref. [23], for the value of $U=4 \mathrm{eV}$ which is about twice as big as $U-J=2.1 \mathrm{eV}$ used in our calculations. This explains why their spin moments are slightly bigger.

[45] J. Stohr and H. Konig, Phys. Rev. Lett. 75, 3748 (1995).

[46] G. van der Laan, J. Phys.: Condens. Matter 10, 3239 (1998).

[47] J. Trygg, B. Johansson, O. Eriksson, and J. M. Wills, Phys. Rev. Lett. 75, 2871 (1995).

[48] A. B. Shick and W. E. Pickett, Phys. Rev. Lett. 86, 300 (2001).

[49] A. B. Shick, V. Drchal, and L. Havela, Europhys. Lett. 69, 588 (2005).

[50] M. Cococcioni and S. de Gironcoli, Phys. Rev. B 71, 035105 (2005). 